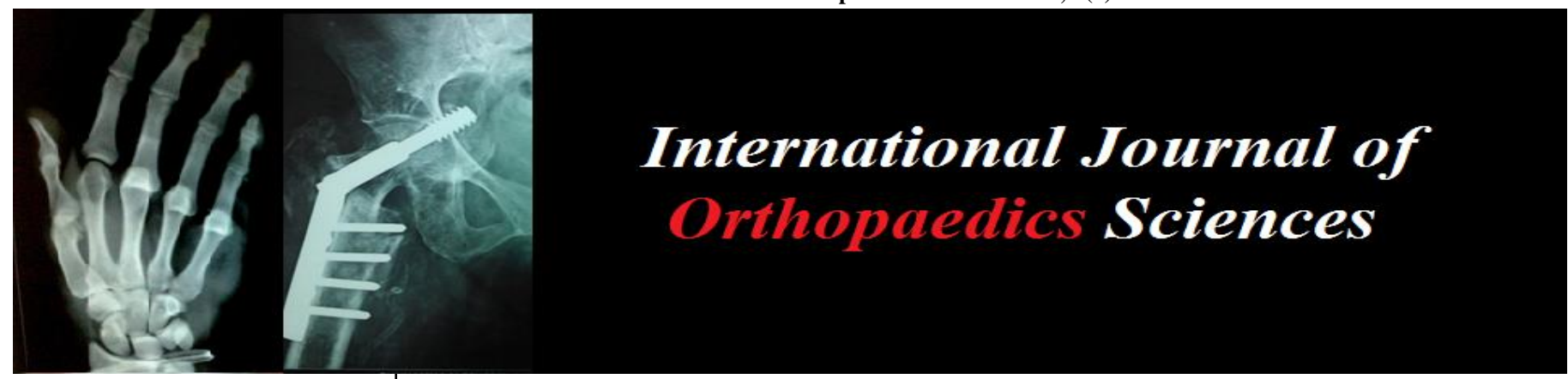

ISSN: $2395-1958$

IJOS 2019; 5(2): 985-990

(C) 2019 IJOS

www.orthopaper.com

Received: 01-02-2019

Accepted: 03-03-2019

Milind Deshpande

Associate Professor,

Department of Orthopedics,

Goa Medical College,

Bambolim, Goa, India

Karthikeyan Shanmugam

Junior Resident, Department of

Orthopedics, Goa medical

College, Bambolim, Goa, India

Kunal Fadte

Senior Resident, Department of

Orthopedics, Goa medical

College, Bambolim, Goa, India

Correspondence

Karthikeyan Shanmugam

Junior Resident, Department of

Orthopedics, Goa medical

College, Bambolim, Goa, India

\section{Proximal femoral nailing-intra operative complications: A retrospective analysis of 200 cases of proximal femoral fractures}

\section{Milind Deshpande, Karthikeyan Shanmugam and Kunal Fadte}

DOI: https://doi.org/10.22271/ortho.2019.v5.i20.1464

\section{Abstract}

Background: Proximal femoral fractures are common in elderly and with high velocity trauma in younger age groups too. Nailing of proximal femoral fractures is technically demanding procedure which needs experience to tackle the difficulties. Detailed literature regarding the difficulties faced during the procedure is lacking.

Methods: Analysis of the intra operative complications faced during closed and open proximal femoral intra-medullary nailing.

Conclusions: Technical difficulties arise due to many reasons starting from improper positioning of the patient. With good instrumentation and with experience these problems can be minimized with attention to each surgical step.

Keywords: Proximal femoral nail, proximal femoral fractures, sub-trochanteric fractures, inter trochanteric fractures

\section{Introduction}

Hip fractures are devastating injuries at any age. Treatment options for proximal femoral fractures include screw-side plate devices and intramedullary nails. Intramedullary nailing is preferred over plates especially in unstable fractures due to their improved biomechanics, smaller incision, less blood loss, decreased post-operative femoral neck varus malunion. Many surgeons still prefer Dynamic hip screw (DHS) plate due to technical difficulties in doing proximal femoral nailing. This is a step wise analysis of factors that can lead to intra operative difficulties in reduction and fixation thereby affecting the outcome of surgery ${ }^{[1]}$.

\section{Methods}

This is a retrospective analysis of intraoperative complications of proximal femoral fractures treated with intra medullary nailing done between 2015-2017 in patients admitted under Orthopedic Department, Goa medical college, Goa, India.

\section{Discussion}

Postioning: Proper positioning of the patient in a banana shape with affected leg and torso adducted is important to give proper room for the surgeon while passing the intra-medullary femoral shaft guide wire, reaming and inserting the nail. This is important especially in obese patients. We prefer to do DHS for morbidly obese patients.

The foot of the affected leg should be secured properly in the boot of the fracture table and perineum should touch the perineal post for effective hip traction.

Sometimes the intertrochanteric fracture pattern may be such that on adduction of the limb the fracture malpositions into varus and traction fails to correct varus and hence the leg has to be abducted ${ }^{[2]}$ to neutral following insertion of the nail in adduction and then cervical screws are put after confirming proper neck shaft angle on $\mathrm{C}$-arm. In neutral position of the limb the proximal jig impinges on the flank, especially in obese patients, that the incision at the entry has to be enlarged significantly to embed and accommodate the jig in the depth of the incised tissues thus increasing chances of infection, increased operative time, blood loss and long scar. 
Excessive traction, in an attempt to reduce fracture, can lead to pelvic rotation around the perineal post and produce relative abduction of the hip, thus interfering with access to the proximal femoral nail (PFN) entry site ${ }^{[3]}$.

\section{Entry}

The entry portal for proximal femoral nailing is a modified medial trochanteric portal ${ }^{[4-6]}$.

This is located at the medial aspect of the greater trochanter along the trochanteric ridge on the antero-posterior view and in line with the femoral medullary canal in the lateral view on $\mathrm{C}$-arm imaging. Comminution at the greater trochanter will cause difficulty in creating a proper entry. A lateral starting portal will lead to varus malalignment. If such a portal is causing malalignment, it can be corrected by placing a small plate within the reamed lateral tract and re-reaming a more medial portal or by using the original portal and adding an antero-posterior blocking screw to correct the malalignment. When the entry portal is inadvertently posterior the placement of neck screws occurs in two different planes since the nail travels from proximal posterior to distal anterior especially when the nail diameter is less than the medullary canal diameter.

The greater trochanter can fracture while making the entry portal in osteoporotics or sometimes an undisplaced fracture not seen on the pre-op $\mathrm{x}$-ray or on the $\mathrm{C}$-arm can displace.

\section{Sub-trochanteric fractures}

It's difficult to make a proper entry portal in the greater trochanter in sub-trochanteric fractures especially when the proximal fragment is significantly flexed. Initially we used to make an entry point in the flexed proximal femur fragment in the direction of themedullary canal and then inserted a cannulated rigid reamer into it to manipulate it and reduce the fracture and then pass the guide wire into the distal fragment through the reamer and then ream the distal fragment. However, the reduction could not be maintained effectively with a guide wire in-situ while trying to change reamers. The error in the entry point due to a flexed abducted and externally rotated proximal fragment and difficulty in maintaining reduction with guide wire in-situ during reaming, all lead to a malreduction and eventually to malunion or nonunion at times.

In Fielding type $3^{[7]}$ transverse sub-trochanteric fractures we made an incision just enough for the entry portal and to put a bone holder in the high sub-trochanteric region to manipulate the proximal fragment rather than doing an open reduction of the fracture. We routinely did open reduction for high subtrochanteric fractures.

During closed nailing of sub-trochanteric fractures on fracture table in supine position the leg is kept in neutral position to prevent the distal fragment going into further adduction and this may cause the jig to impinge on the flank necessitating long incisions and it also thus mandates the use of flexible reamers which in turn require the non beaded guide wires to be exchanged with beaded ones thus increasing operative time as compared to using non-beaded guide wires and cannulated rigid reamers over them.

When flexible reamers are used, sub-trochanteric fractures have to be manually reduced with pressure on the anterior thigh while reaming across the fracture site. If reaming is continued without fracture reduction, the reamers tend to damage the cortices of the distal fragment and hence comminution at fracture site.

\section{Femur shaft with neck/intertrochanteric fracture}

In cases with fracture of neck or inter trochanteric fracture with shaft of femur fracture, it is technically demanding to get proper reduction of both fractures using one implant i.e., PFN. The proximal femur fracture is fixed first and then the shaft fracture is addressed before locking the nail distally. During fixation with a PFN some amount of malreduction may be accepted in an inter-trochanteric fracture reduction but the reduction of fracture neck has to be perfect even if open reduction is required. Hence we often prefer fixation of neck shaft fracture combination with two different implants ${ }^{[8]}$.

\section{Guide Wire}

After the entry point is made with an awl, we routinely insert a non-beaded guide wire into femoral canal and ream with cannulated rigid reamers. In two cases where we had used cannulated flexible reamers we had the following complications.

1. In the first case, we forgot to exchange the beaded guide wire witha non-beaded guide wire. Then a 10size nail was inserted and we drilled the cervical guide wires into head neck fragment with great difficulty. The cervical guide wires managed to clear the nail to enter the femoral neck. Then on attempting to drill with cannulated drill bits across the nail we realized the gross error of not removing the femoral guide wire. The beaded guide wire couldn't be removed on attempting as the beaded tip couldn't navigate through the nail. The entire steps had to be revised.

2. In the second case, a similar situation occurred where a $13 \mathrm{~mm}$ nail was inserted with a beaded guide wire in-situ. However the error could be corrected since the beaded guide wire came out through the nail as it was a $13 \mathrm{~mm}$ diameter nail.

\section{Nailing}

Two complications are faced if the femur is bowed excessively in the sagittal plane.

1. The distal end of the nail may abut the anterior cortex of the femur and may cause chronic anterior thigh pain on weight bearing and may also work as a stress riser especially in cases with osteoporosis ${ }^{[9-13]}$.

2. In one case we had an intra operative complication of the distal end of the short proximal femoral nail $(250 \mathrm{~mm}$ nail length) penetrating the anterior femoral cortex and causing a complete shaft fracture. The fixation was revised with a long nail ${ }^{[15-17]}$.

If excess bowing is noted the trochanteric femoral nail (TFN) [Figure1a-b] (140-150mm nail length) can be used instead of short PFN (230-250mm).

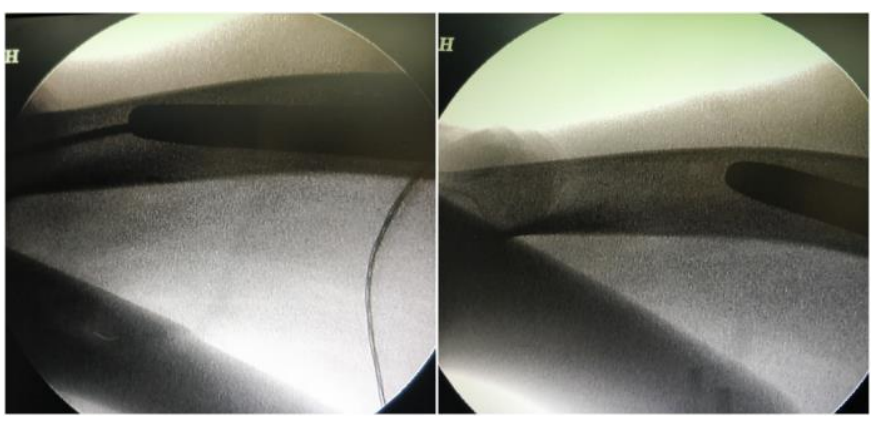

Fig 1a-b: Radiographs showing short PFN hitting the anterior cortex[a] replaced with TFN[b] 
A slight anterior trochanteric entry portal [Figure2a-b] can be made so that the nail does not hit the anterior cortex in such cases.
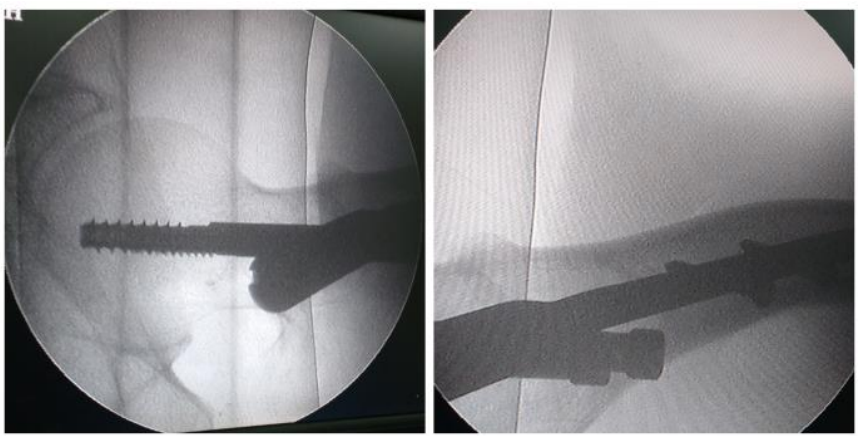

Fig 2a-b: Radiographs showing anterior entry portal[a] made in bowed femur[b] to negotiate the short PFN

\section{Loss of Reduction}

A. When inserting the proximal broader portion of the nail, it may cause loss of reduction by pushing the proximal fragment of an intertrochanteric fracture into varus. The nail can also distract the fracture by pushing the distal fragment laterally. Both these situations occur since either or both fracture fragments have not been reamed properly with the proximal nail diameter reamer. The following three methods can be used during reaming and inserting the nail to prevent this:

1. The fracture fragments have to be reduced and compressed by a medially directed force applied to the lateral trochanteric region.

2. Another method is by removing a small cortical bone from the superolateral edge of the medial fragment at the site of entry portal ${ }^{[2]}$.

3. The problem can be minimized by over reaming the proximal femur till the sub-trochanteric region. Solid reamers can actually push the distal fragment laterally without reaming it at the entry and fracture site and hence flexible reamers may have to be used in such situations.

B. When inserting the proximal broader portion of the nail, it may cause loss of reduction by translating the proximal fragment distally and this can be prevented by using a long artery forceps on the inferior portion of the neck to push the head neck fragment proximally ${ }^{[2]}$.

\section{Greater Trochanter Fracture}

Diameter of the proximal part of the nail is $16-17 \mathrm{~mm}$ in PFN. This is relatively large for Indian population and small built females and thus may lead to iatrogenic greater trochanter fracture during reaming or nail insertion ${ }^{[6]}$. Now some Indian companies are manufacturing $\mathrm{PFN}$ with proximal nail diameter of $15 \mathrm{~mm}$.

\section{Unstable Inter-Trochanteric Fracture with Sag}

In unstable fractures with posterior sag the distal fragment is lifted holding the proximal jig and the cervical guide wires are passed and this position has to be held accurately till the procedure of reaming and inserting screws is over and if the assistant relaxes, the sag recurs and the guide wires provided by certain implant manufacturers $(2 \mathrm{~mm})$ bend at the fracture site and then when the sag is recorrected and reaming started, the reamer will have some difficulty to run across the deformed guide wire and because of this guide wires can break at deformed point when the reamer reaches that point and reaming is continued hoping to run across.
At times we lower the foot end of the affected limb in unstable fracture with sag so that we need not lift and hold the proximal jig too much and for too long. The sag is corrected by a broad bone lever placed posterior to the fracture when the technique of correcting sag by lifting the proximal jig fails and the failure is due to poor inter digitation of fracture leading to lifting of only distal fragment by the jig. At times the jig technique works if the jig is pressed medially thus inter digitating the fracture and then lifting it. The advantage of the jig technique is the guide wires can be visualized for their positioning on c-arm from their entry point in the lateral femoral cortex while this is not possible when using the radio opaque broad lever technique. The use of radio lucent levers/retractors is mentioned while doing the high tibial osteotomy but we do not posses them ${ }^{[18]}$.

\section{Jig Nail Assembly}

Assembling the nail on to the appropriate jig is important for matching of the screw slots of the nail and jig. Assembling a $130^{\circ}$ nail on to a $135^{\circ}$ jig or vice versa will lead to mismatch. Jig nail assembly is checked with sleeves, guide wires and reamers before the nail is inserted. This mix up occurs when both $130^{\circ}, 135^{\circ}$ nails and jigs are autoclaved and presented in the same box at surgery.

\section{Cervical Guide Wires}

Inserting 2 cervical guide wires precisely in the head and neck fragment is difficult in patients with a small diameter head and neck, which we encountered in short statured patients, especially females. In some patients screws were accommodated with great difficulty and in some patients we could put only the inferior calcar screw [Figure3a-b] ${ }^{[6,14]}$.

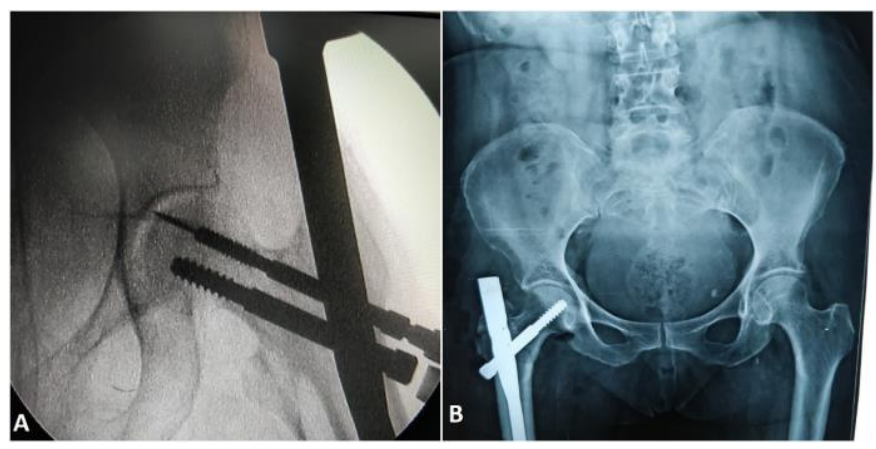

Fig 3A-B: Radiographs showing small neck where both screws are very close to cortex $[\mathrm{A}]$ and another case where only one screw could be accommodated[B]

The two screws $(8 \mathrm{~mm}$ inferior/calcar screw $+6 \mathrm{~mm}$ derotation screw) with distance of $10 \mathrm{~mm}$ between them in the nail necessitates the minimum size of the neck to be $35 \mathrm{~mm}$ in coronal plane (superior cortex to inferior cortex) for insertion of both cervical screws[Figure4a-b].

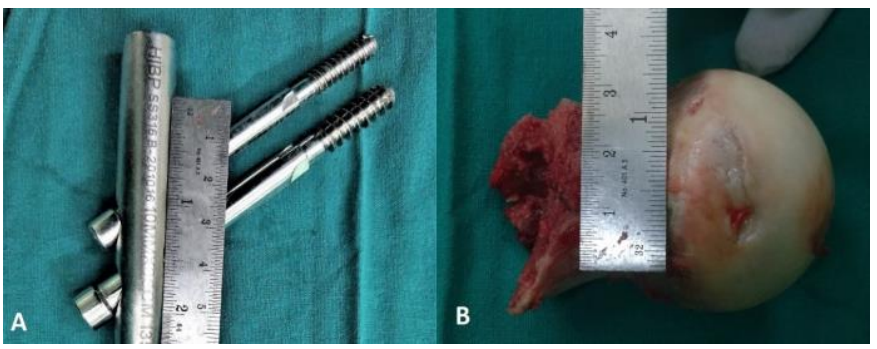

Fig 4a-b: Distance between the two cervical screws [a] and Specimen of Indian female with short neck $(25 \mathrm{~mm})[\mathrm{b}]$ 


\section{Neck Shaft Angle}

The nails available to us are of only 2 angles i.e., $130^{\circ}$ and $135^{\circ}$ and hence it is not possible to insert 2 cervical screws in patients with neck-shaft angle of less than $130^{\circ}$. The inferior guide wire travels from inferior quadrant in base of neck to superior quadrant in head when a $130^{\circ}$ or $135^{\circ} \mathrm{jig}$ is used in patients with neck shaft angle of less than $130^{\circ}$ and we often encounter this in Indian population.

\section{Bending, Breakage of Cervical Guide Wires}

The guide wires can bend inside the neck during insertion especially in patients with good bone stock, due to their thin diameter. The guide wires can also bend in strong subchondral bone of the femoral head and reaming over them has resulted in their breakage at the terminal $1 \mathrm{~cm}$ of the guide wire and hence we stop the reaming at the bent site, withdraw the guide wire completely and then ream the subchondral portion without guide wire.

In unstable and osteoporotic fractures, the guide wires often get stuck inside the reamer and come out with the reamer when the reamer is being withdrawn and hence we started transfixing the guide wires to the acetabulum until we had our complication of guide wire breakage in subchondral bone of femoral head during reaming. The broken wire across hip joint was removed by reaming over it into the acetabulum very slowly watching on c-arm for dislodgement of the broken wire into the pelvis which luckily didn't happen and the wire got stuck in the reamer and came out. We now never transfix the guide wire into acetabulum.

\section{Guide wire advancement in head of femur}

The guide wire must be advanced just below the femoral head margin after measuring the screw length required when guide wires have reached the correct tip -apex distance. So even if the guide wires come out on reaming they can be replaced in the correct position through their terminal tract in femoral head which matches their diameter. If the guide wires are placed only upto correct tip-apex distance and if reaming done over them causes them to come out, replacing them in the reamed canal becomes inaccurate leading to placement of the screws in a slightly different newer channel created by the screw itself in the osteoporotic bone and thus leading to placement of a screw in a wider hole in which it can toggle and loosen.

\section{Guide wires not parallel}

Guide wires may not be parallel on antero-posterior (AP) view in the neck [Figure.5] due to bending in strong subchondral bone or due to mal-reduction. On the lateral view the guide wires will not be parallel if the entry point of the nail is posterior.

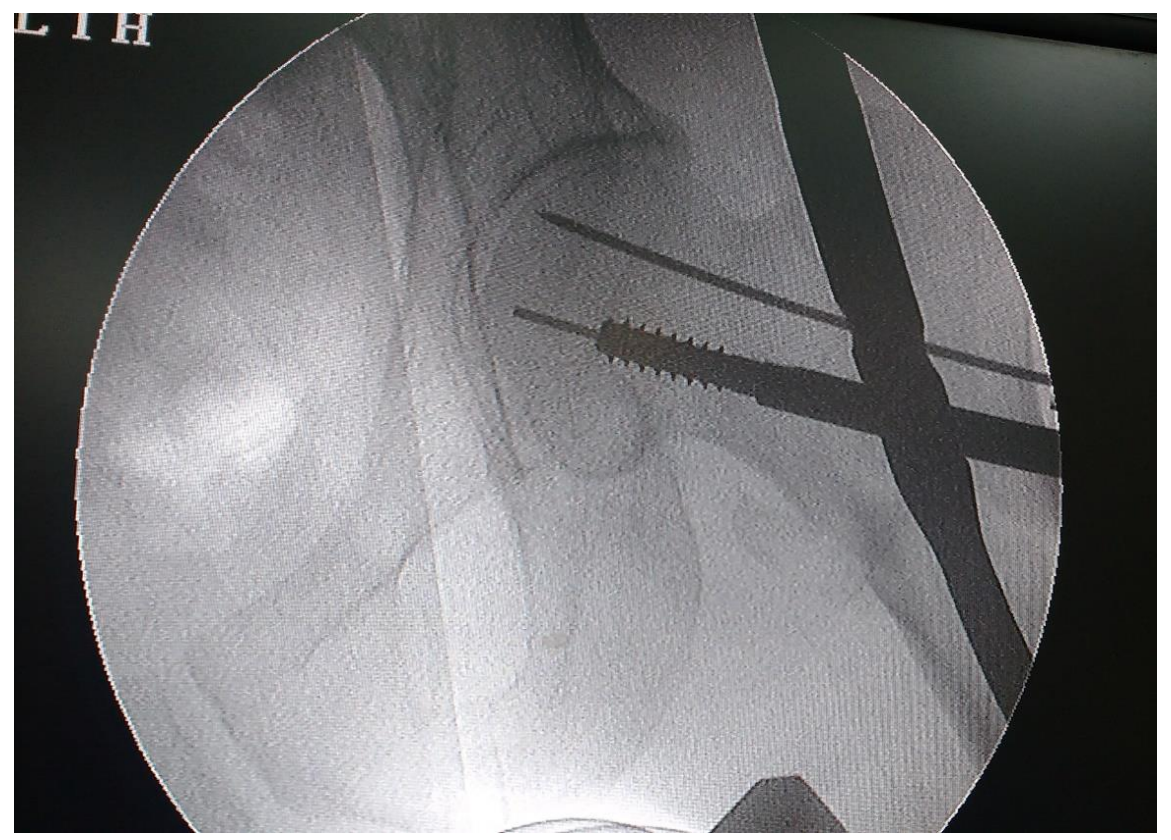

Fig 5: Radiograph showing bent guide wire in the neck of femur

\section{Overuse of instrumentation}

The sleeve inserted through the jig is sometimes impacted with hammer to make sure that it touches the lateral femoral cortex. This may deform the sleeve tip. In one case the neck was drilled for the screw but when the screw was inserted the screw head was not negotiating through the sleeve and hence the screw and sleeve were removed only to find sleeve tip deformed and not allowing screw head to negotiate further.

\section{JIG and nail mismatch}

In one case, the guide wires were not parallel in the neck since the proximal guide wire was bent. Here the distal $8 \mathrm{~mm}$ screw was inserted first and then we tried to ream for the proximal screw. But the reamer was hitting the nail and not getting into the screw hole and hence we removed the guide wire and reamed without sleeve under $\mathrm{C}$-arm guidance and transfixed guide wire through the reamer and removed the reamer followed by inserting proximal screw [Figure.6]. This happened even though the jig sleeve nail assembly was checked for their matching before inserting the nail. At times we had to make a lateral cortical window to ream and insert screws in cases of jig nail mismatch. The mismatch probably occurs in situ due to the nail bending in the femur and this conclusion was supported by the fact that no mismatch was evident upon removing the nail and checking for mismatch in vitro. 


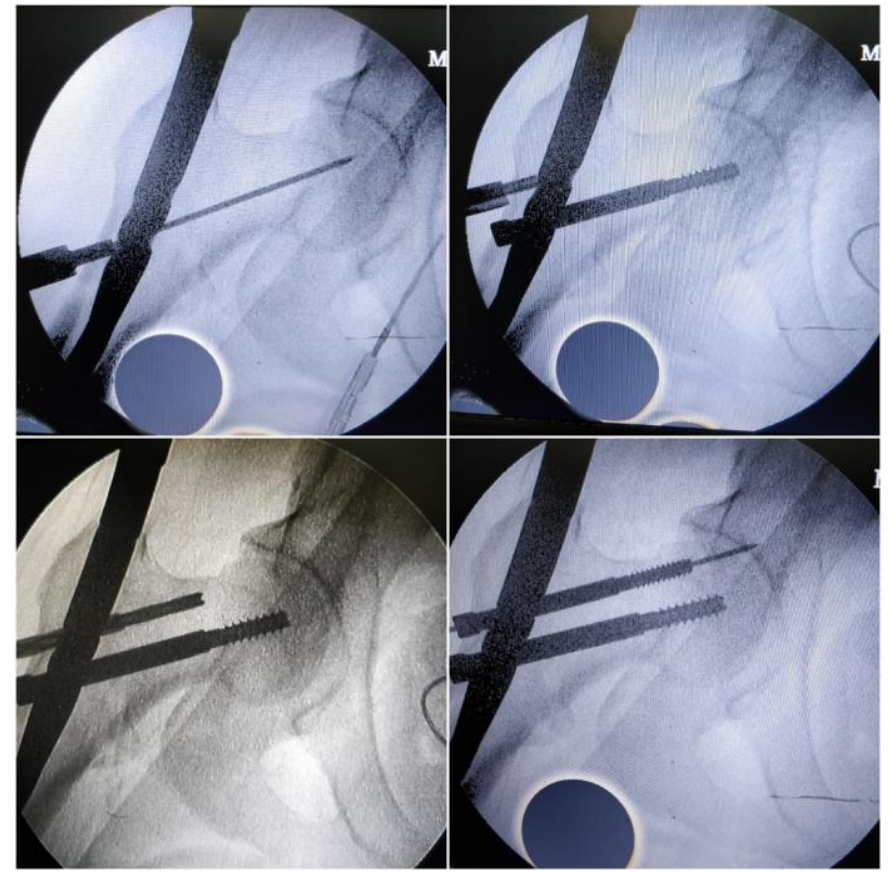

Fig 6a-b: Guide wire going through the superior part of the slot (a). Drill bit not matching with the slot (b). Drilling done without sleeve (c). Guide wire inserted through the drilled hole and screw inserted (d)

\section{PFN spiral blade}

Proximal femoral nailing and fixation with a spiral blade involves gentle hammering of the blade across the intertrochanteric fracture site. In one case, the greater trochanter fractured proximal to the spiral blade entry site during hammering, since the bone was osteoporotic [Figure7]. This risk remains in all osteoporotic patients.

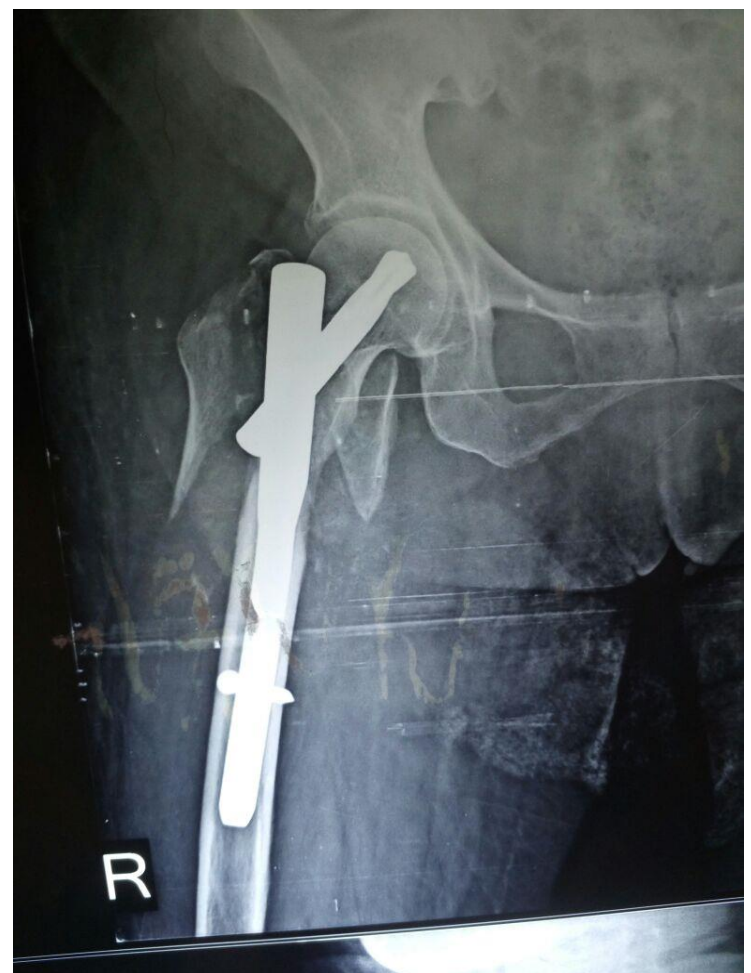

Fig 7: Radiograph showing greater trochanter comminution
In another case, the spiral blade was inserted after proper reduction was achieved with the initial position of the leg in adduction on fracture table and when the leg was abducted for distal locking the fracture distracted. This might have happened due to lack of good hold of the blade in the head of femur probably because of the lack of threads [Figure8].

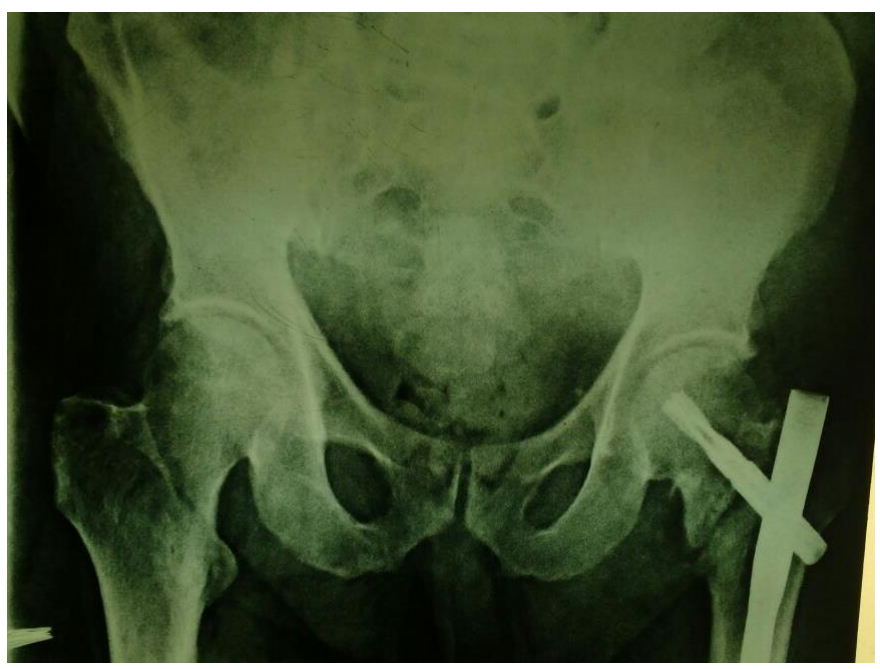

Fig 8: Radiograph showing distraction at fracture in spiral blade fixation

\section{Radio-Opague Jig}

If the cervical guide wires are placed in the central quadrant, in the lateral view the guide wires cannot be visualized many a times on $\mathrm{C}$-arm image since the proximal jig is radio-opaque and overlaps them and hence the wires are placed in slight ante-version so that they can be seen on the lateral view on carm. If the inter trochanteric fracture is stable it is easy to maintain the ante-version of the proximal fragment and the cervical guide wires can be seen in the lateral view even if they are in the central quadrant. In unstable fractures where it is difficult to maintain the ante-version, the guide wires have to be passed in centero-anterior quadrant such that the horizontal arm of the radio-opaque jig ${ }^{[18]}$ does not come in way of visualizing the guide wires.

In cases with postero-medial comminution, the distal fragment was externally rotated to close the comminution. In such cases the ante-version was $0^{\circ}$ and hence the guide wires had to be passed into the centero-anterior quadrant to visualize them in lateral view. In some cases we accepted not to visualize the cervical-guide wire in lateral view when they were placed in the central quadrant and relied upon seeing them within the boundaries of the radio-opaque shadow of the jig after we penetrated them into the hip joint to confirm the position and later withdrew them proximally before drilling over them.

Jig being radio-opaque besides being defective can also be a possible reason for the reported complication of proximal screws missing the nail in the lateral view.

\section{Proud Proximal Nail}

The proximal tip of the nail remained proud from the greater trochanter in patients with short stature who had a small greater trochanter thus later causing pain and bursitis especially in thinly built patients [Figure3b] ${ }^{[19]}$. 


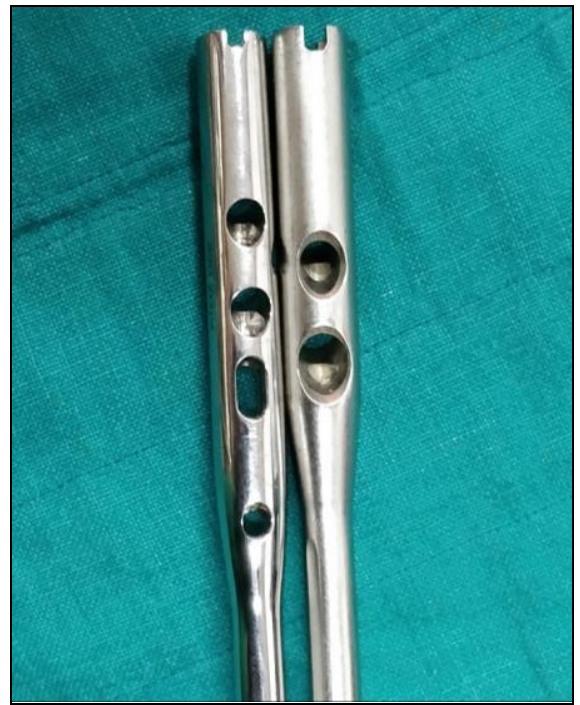

Fig 9: Nails from different manufacturers with different length of nail segment proximal to cervical screw slots

Conflict of Interest: The authors declare that they have no conflict of interest.

Funding: There is no funding source.

Ethical approval: This article does not contain any studies with human participants or animals performed by any of the authors.

\section{References}

1. Pichler W, Mazzurana P, Clement H et al. Frequency of Instrument Breakage during Orthopaedic Procedures and Its Effects on Patients. J Bone Jt Surgery-American. 2008; 90:2652-2654. doi: 10.2106/JBJS.H.00163

2. Aithala PJ, Rao S. Proximal Femoral Nailing: Technical Difficulties and Results in Trochanteric Fractures. Open J Orthop. 2013; 03:234-242. doi: 10.4236/ojo.2013.35044

3. Proximal femur - Reduction \&amp; Fixation - Closed reduction; short intramedullary nail (PFNA) - A1 - AO Surgery Reference.

https://www2.aofoundation.org/wps/portal/!ut/p/a1/04_Sj 9CPykssy0xPLMnMz0vMAfGjzOKN_A0M3D2DDbz9_ UMMDRyDXQ3dw9wMDAzMjYEKIvEocDQnTr8BDu BoQEh_QW5oKAD4ENaS/dl5/d5/L2dJQSEvUUt3QS80 SmlFL1o2XzJPMDBHSVMwS09PVDEwQVNFMUdW RjAwMDcz/?showPage=redfix\&bone=Femur\&segment. Accessed 19 Jul 2018

4. Green R. and Fractures in adults. In: 8th ed vol.2. p chapter 52,2174-2180

5. Sheth U, Gohal C, Chahal J et al. Comparing Entry Points for Antegrade Nailing of Femoral Shaft Fractures. Orthopedics. 2016; 39:e43-e50. doi: 10.3928/0147744720151218-09

6. Kanthimathi B, Narayanan V. Early Complications in Proximal Femoral Nailing Done for Treatment of Subtrochanteric Fractures. Malaysian Orthop J. 2012; 6:25-29. Doi: 10.5704/MOJ.1203.009.

7. Koval KJ, Zuckerman JD, Joseph D. Hip fractures : a practical guide to management. Springer, 2000.

8. Robert W Bucholz, James D Heckman, Charles M CourtBrown, Paul Tornetta. III. Rockwood and Green's Fractures in Adults. Seventh Edition. Philadelphia: Lippincott Williams and Wilkins, 2010.

9. Egol KA, Chang EY, Cvitkovic J et al. Mismatch of current intramedullary nails with the anterior bow of the femur. J Orthop Trauma, 2004. doi: 10.1097/00005131200408000-00003

10. Maratt J, Schilling PL, Holcombe S et al. Variation in the Femoral Bow. J Orthop Trauma. 2014; 28:6-9. doi: 10.1097/BOT.0b013e31829ff3c9

11. Chang S-M, Hu S-J, Ma Z et al. Femoral intertrochanteric nail (fitn): a new short version design with an anterior curvature and a geometric match study using post-operative radiographs. Injury. 2018; 49:328333. doi: 10.1016/j.injury.2017.11.017

12. Walensky NA. A study of anterior femoral curvature in man. Anat Rec. 1965; 151:559-570. DOI: 10.1002/ar.1091510406.

13. Pathrot D, Ul Haq R, Aggarwal AN et al. Assessment of the geometry of proximal femur for short cephalomedullary nail placement: An observational study in dry femora and living subjects. Indian J Orthop. 2016; 50:269-76. doi: 10.4103/0019-5413.181785

14. Sengodan VC, Sinmayanantham E, Kumar JS. Anthropometric analysis of the hip joint in South Indian population using computed tomography. Indian J Orthop. 2017; 51:155-161. doi: 10.4103/0019-5413.201709

15. Kanawati AJ, Jang B, McGee R, Sungaran J. The influence of entry point and radius of curvature on femoral intramedullary nail position in the distal femur. $\mathbf{J}$ Orthop. 2014; 11:68-71. doi: 10.1016/j.jor.2014.04.010.

16. Roberts JW, Libet LA, Wolinsky PR. Who is in danger? Impingement and penetration of the anterior cortex of the distal femur during intramedullary nailing of proximal femur fractures. J Trauma Acute Care Surg. 2012; 73:249-254. doi: 10.1097/TA.0b013e318256a0b6

17. Bazylewicz DB, Egol KA, Koval KJ. Cortical Encroachment after Cephalomedullary Nailing of the Proximal Femur. J Orthop Trauma. 2013; 27:303-307. doi: 10.1097/BOT.0b013e318283f24f

18. Gadegone W, Lokhande V, Salphale Y, Ramteke A. Long proximal femoral nail in ipsilateral fractures proximal femur and shaft of femur. Indian $\mathbf{J}$ Orthop. 2013; 47:272-7. doi: 10.4103/0019-5413.109441

19. Hu SJ, Chang SM, Ma Z et al. PFNA-II protrusion over the greater trochanter in the Asian population used in proximal femoral fractures. Indian J Orthop. 2016; 50:641. doi: 10.4103/0019-5413.193475 